\title{
Análisis de las demoras en salud en personas que enfermaron de gravedad o fallecieron por dengue en cinco ciudades de Colombia
}

I 1 Fabián Ardila Pinto, ${ }^{2}$ Sandra Martínez, ${ }^{3}$ Mauricio Fuentes, ${ }^{4}$ Elizabeth Borrero I

Resumen: Objetivo: Identificar demoras en la atención médica de personas que fallecieron o se recuperaron de dengue grave en cinco ciudades de Colombia. Materiales y métodos. Estudio cualitativo sobre personas que fallecieron por dengue (casos) o que se recuperaron (controles). Se entrevistó a los familiares y se analizaron sus itinerarios de búsqueda de atención en salud. Los datos fueron analizados desde el modelo de las "tres demoras", complementado con diagramas espacio-temporales y entrevistas a profesionales de salud. Resultados: A pesar de desconocer signos y síntomas, se buscó atención rápidamente. Para acceder a la primera consulta el recorrido no representó dificultades. Se presentaron fallas en la calidad del diagnóstico y un mayor número de instituciones consultadas en los casos que en los controles. Se evidenció un proceso de retorno a la demora 1 que puede influir negativamente en futuras decisiones en salud. Conclusiones:

Complementar el análisis clásico de demoras con el análisis espacio-temporal de los itinerarios permite visibilizar fallas y aciertos en la calidad de la atención y aportar a la toma de decisiones.

> Palabras-clave: dengue; atención médica; mortalidad; análisis cualitativo.

\author{
1 Fundación Santa Fe de \\ Bogotá. Centro de Estudios e \\ Investigación en Salud. \\ Bogotá, Colombia. Endereço \\ Correo electrónico: quiliquen@ \\ gmail.com \\ 2 Fundación Santa Fe de \\ Bogotá. Centro de Estudios e \\ Investigación en Salud. \\ Bogotá, Colombia. Endereço \\ Correo electrónico: \\ sanmart79@gmail.com \\ ${ }^{3}$ Fundación Santa Fe de \\ Bogotá. Centro de Estudios e \\ Investigación en Salud. \\ Bogotá, Colombia. Endereço \\ Correo electrónico: cus_mf@ \\ hotmail.com \\ ${ }^{4}$ Fundación Santa Fe de \\ Bogotá. Centro de Estudios e \\ Investigación en Salud. Bogotá, \\ Colombia. Endereço Correo \\ electrónico: elizabeth.borrero@ \\ fsfb.org.co
}

Recibido: 23/07/2014

Aprobado: 18/12/2014 


\section{Introducción}

El dengue es un problema de salud pública por su magnitud e impacto económico en el sistema de salud y las familias (PADILLA, 2012). Se trata de una enfermedad infecciosa sistémica y dinámica que puede cursar de forma asintomática, o expresarse en forma grave o no grave (OPAS, 2010). En el mundo se presentan 50 millones de infecciones anualmente. En la región de las Américas se ha producido un incremento progresivo de casos de dengue durante las tres últimas décadas extendiéndose a casi todos los países (MARTÍNEZ TORRES, 2008).

En Colombia, se estima que existen alrededor de 23 millones de personas en riesgo especialmente en las cabeceras municipales. La enfermedad ha presentado una tendencia ascendente, especialmente en los últimos 15 años, así, durante el periodo 1978-2010, se registró oficialmente en el país un acumulado de 1,020,637 casos de dengue (PADILLA, 2012).

Para entender el proceso enfermedad- atención- recuperación o muerte, ha sido de gran utilidad el modelo "camino a la supervivencia" o de las 3 demoras: 1) demora en decidir buscar atención, 2) demora en llegar a los servicios de salud y 3) demora en recibir el tratamiento adecuado (THADDEUS; MAINE, 1997). Estas autoras usan el concepto de "demoras" como el periodo entre el inicio de una complicación hasta su adecuado tratamiento y su relación con factores como la autonomía para buscar asistencia médica, la distancia y los servicios de salud (PACAGNELLA; CECATTI 2012). Si bien esta metodología fue diseñada para el análisis de la mortalidad materna, permite identificar y clasificar las barreras y situaciones relacionadas con la búsqueda de atención en salud de familias y personas ante el evento de enfermar o morir.

El objetivo del estudio fue identificar las demoras e itinerarios en la atención médica de las personas que fallecieron por dengue, o que se recuperaron de dengue grave en cinco ciudades de Colombia, construyendo una relación entre el análisis de demoras y los itinerarios de las personas a través de metodologías cualitativas y el análisis geográfico.

\section{Materiales y métodos}

A partir de un estudio epidemiológico de casos y controles se identificaron personas que fallecieron por dengue (casos), así como personas que se recuperaron después de un dengue grave (controles). Se seleccionó una submuestra de 13 casos 
y 8 controles para profundizar por medio de entrevistas semiestructuradas sobre

las tres demoras en el proceso de búsqueda de atención (MAINE, 1997) y 1994. Esta submuestra fue seleccionada teniendo en cuenta los siguientes criterios: 1) que las personas procedieran de las ciudades seleccionadas en el estudio, 2) que contaran con diagnóstico confirmado para la enfermedad a través de la previa revisión de historia clínica (para los casos Reacción en cadena de la polimerasa -PCR- positiva para dengue y para controles IgM o NS1 positiva) y 3) aceptar participar a través de la firma de consentimiento informado.

Una vez la persona cumplía con los criterios de inclusión, se programó una visita al hogar o lugar acordado, y posteriormente se llevó a cabo el encuentro para realizar el proceso de entrevista.

Se diseñó un instrumento para indagar sobre los siguientes aspectos: información sociodemográfica, antecedentes y conocimientos previos sobre la enfermedad, reconocimiento del problema, recorrido para la búsqueda de atención, atención recibida en la (s) institución (es) de salud, percepción de la atención recibida, experiencias posteriores a la enfermedad (muerte o recuperación). Este instrumento fue validado en dos ciudades, previamente a la aplicación con las personas incluidas en el estudio.

Se llevaron a cabo entrevistas a los familiares más cercanos de los casos y en cuanto a los controles se entrevistó a la persona o un familiar en caso de ser menor de 18 años. Se indagó sobre las demoras en salud desde el inicio de síntomas, generalmente en su domicilio hasta el lugar de atención donde ocurrió el fallecimiento o la recuperación. La recolección de información se realizó en las ciudades de Bucaramanga, Cali, Cúcuta, Girardot y Tame, durante el año 2013.

Para complementar el análisis, se realizaron 16 entrevistas a profesionales de la salud encargados del manejo de la patología en las 5 ciudades (médicos generales, personal de laboratorio, profesionales de vigilancia epidemiológica, médicos internistas e intensivistas), donde se enfatizó en la tercera demora, e indagó por las experiencias de los profesionales de la salud en el sistema, en aspectos como capacitación, pruebas diagnósticas, notificación de febriles, relación médicopaciente, empleo y procedimientos médicos en la atención para dengue.

Todas las entrevistas contaron con consentimiento informado, fueron grabadas, transcritas y procesadas en matrices analíticas donde se tuvo como base la teoría fundamentada, cuyo método permitió abarcar los sucesos y situaciones 
de estudio desde la vivencia de las personas. Posteriormente se realizó una codificación abierta donde se dividieron los sucesos en categorías y subcategorías, buscando mayor oportunidad de descubrimiento (BELTRAMIRO, 2004). Luego se realizó el análisis y triangulación de la información obtenida de las entrevistas, junto con el análisis desde la geografía del tiempo, buscando la inclusión de categorías emergentes y transversales al análisis, hasta conseguir la saturación teórica de los datos. En la tabla 1 se resume el proceso de categorización realizado para llevar a cabo el análisis cualitativo de las demoras en dengue.

\section{Tabla 1. Categorización análisis cualitativo de demoras en dengue}

\begin{tabular}{|c|c|c|c|}
\hline \multicolumn{4}{|c|}{$\begin{array}{c}\text { DEMORAS EN SALUD (CASOS Y CONTROLES) } \\
\text { CATEGORÍAS }\end{array}$} \\
\hline 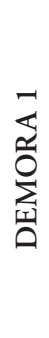 & $\begin{array}{l}\text { Relacionada con tomar la } \\
\text { decisión de buscar atención } \\
\text { en salud. }\end{array}$ & 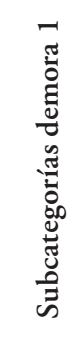 & $\begin{array}{l}\text { - Información sobre el dengue y } \\
\text { - Seconocimiento institucional. } \\
\text { - Inicio de los síntomas. } \\
\text { - Automedicación, género y cuidado } \\
\text { inicial. } \\
\text { - Motivaciones para tomar la decisión } \\
\text { - Redes de apoyo. }\end{array}$ \\
\hline 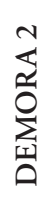 & $\begin{array}{l}\text { Relacionada con todos } \\
\text { aquellos factores y recorridos } \\
\text { una vez se tomó la decisión } \\
\text { de buscar atención en los } \\
\text { servicios de salud. }\end{array}$ & 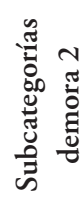 & $\begin{array}{l}\text { - Tiempo de recorrido. } \\
\text { - Sistema de transporte utilizado. } \\
\text { Impacto socioeconómico de la } \\
\text { demora. }\end{array}$ \\
\hline$\sum_{\substack{1 \\
0}}^{\infty}$ & $\begin{array}{l}\text { - Categoría más amplia por } \\
\text { ser el sistema de salud el foco } \\
\text { del análisis. } \\
\text { - Tiene que ver con las } \\
\text { experiencias de atención } \\
\text { en la institución de salud, } \\
\text { desde el momento en que la } \\
\text { persona asume una posición } \\
\text { de paciente o usuario. }\end{array}$ & 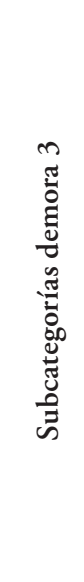 & $\begin{array}{l}\text { - Percepción de la atención en las } \\
\text { instituciones de salud en las cuales } \\
\text { buscó atención. } \\
\text { - Recorridos y experiencias entre las } \\
\text { instituciones. } \\
\text { - Razones del personal de salud para } \\
\text { hacer regresar a las personas a la } \\
\text { vivienda. } \\
\text { - Estrategias familiares. } \\
\text { - Procesos de reconsulta. } \\
\text { - Procesos de remisión dentro del } \\
\text { - } \text { sistema de salud. } \\
\text { - Mortalidad (casos). } \\
\text { - Recuperación (controles). }\end{array}$ \\
\hline
\end{tabular}




\begin{tabular}{|c|}
\hline CATEGORÍAS EMERGENTES \\
\hline $\begin{array}{l}\text { - Retorno a la demora } 1 . \\
\text { - Subcategorías: } \\
\text { - Exequias } \\
\text { - Seguimiento y asesoría institucional post fallecimiento } \\
\text { - Demandas y recursos legales } \\
\text { - Cambio de discurso en nuevos itinerarios en salud. }\end{array}$ \\
\hline CATEGORÍAS TRANSVERSALES \\
\hline $\begin{array}{l}\text { - Empleo (tanto de familias como de profesionales de la salud). } \\
\text { - Derecho a la salud. } \\
\text { - Discurso de los actores. } \\
\text { - Itinerario espacio-temporal. } \\
\text { - Calidad de la atención. }\end{array}$ \\
\hline
\end{tabular}

Se representaron gráficamente los itinerarios en salud utilizando conceptos de la geografía del tiempo (HÄGERSTRAND, 1970). A partir de cada entrevista, se elaboraron diagramas espacio-temporales de los casos y controles graficando sobre un eje X y Y la ubicación de los lugares (hogar e instituciones de salud) y los desplazamientos que se dieron entre estos en el proceso de búsqueda de atención. En el eje X se representó el tiempo transcurrido desde el inicio de síntomas hasta la recuperación según las tres demoras ya mencionadas, y en el eje Y se graficó la distancia, partiendo del entorno más próximo al hogar de la persona hasta un contexto urbano más retirado donde se encuentran servicios especializados de salud. El resultado final fue un diagrama síntesis para los casos y otro para controles, estableciendo las diferencias y particularidades de los itinerarios que contribuyeron a su desenlace (ver figuras 1 y 2).

Para garantizar la confidencialidad, los nombres de las personas entrevistadas que narraron su itinerario, se presentan como casos o controles. No se presentan nombres de Instituciones de salud, EPS (Empresas Promotoras de Salud), ni de las Secretarías de Salud. 


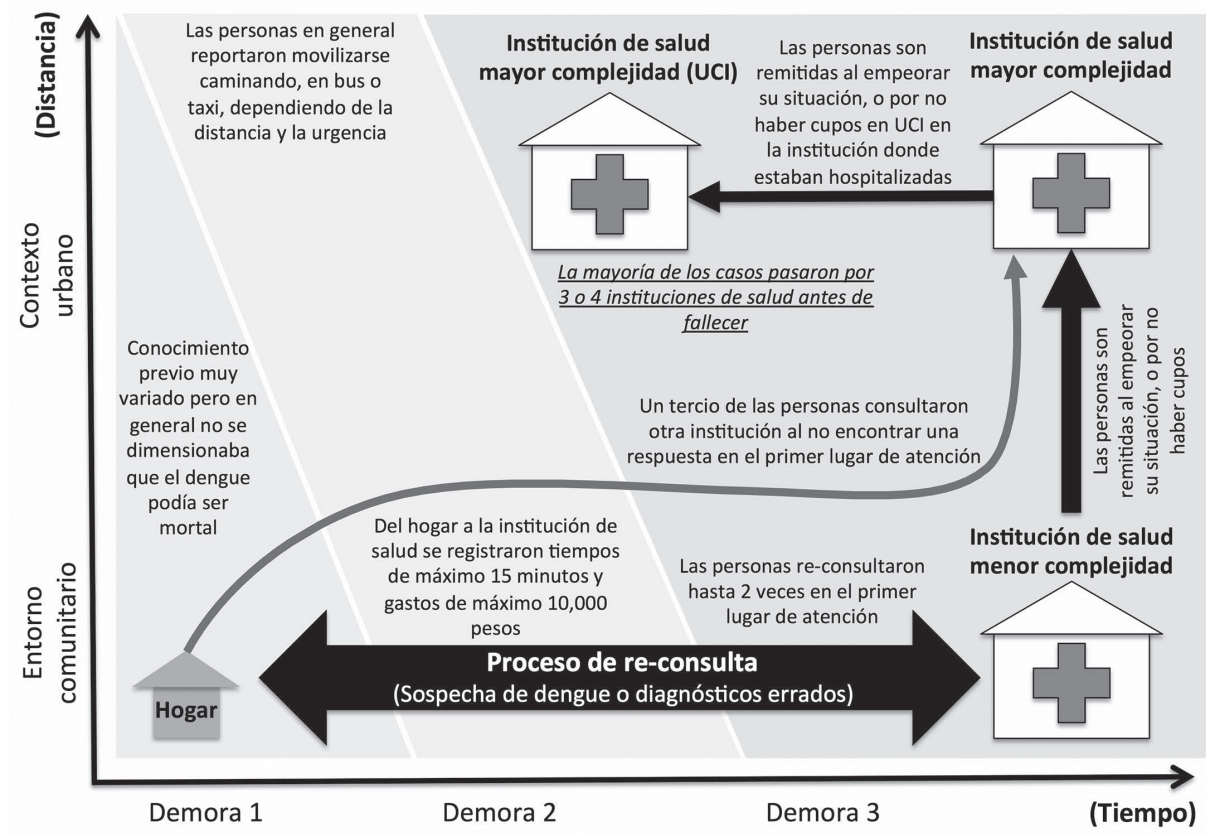

\section{Figura 2}

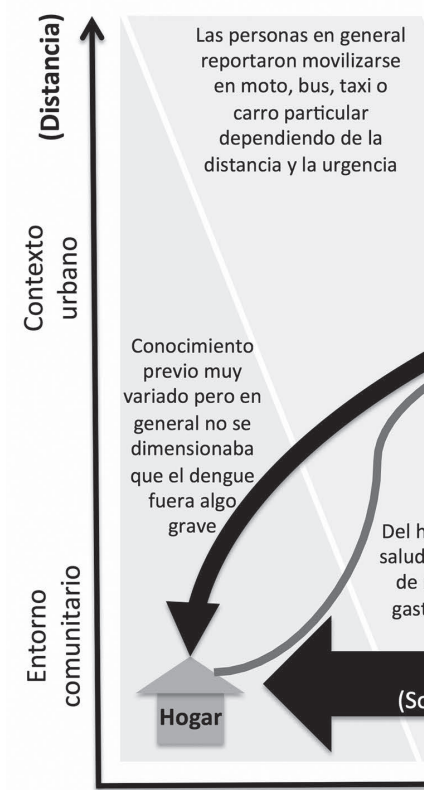

Demora 1

Demora 2

Demora 3

(Tiempo)

Institución de salud Las personas son Institución de salud

la institución donde

Institución de salud lugar de atención

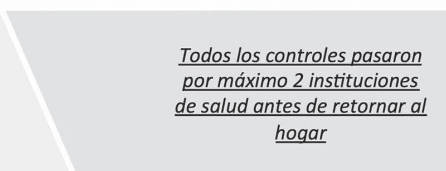

Todos los controles pasaron de salud antes de retornar al hogar

Institución de salud mayor complejidad incluyendo $\mathrm{UCl}$

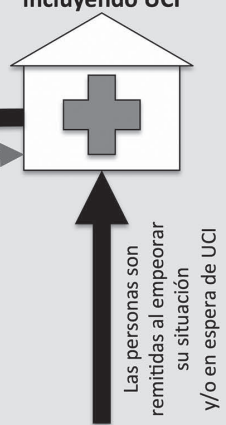

Institución de salud Las personas re-consultaron hasta 2 veces en el primer lugar de atención e máximo 45 minutos $y$ menor complejidad stos de máximo 10,000 pesos Proceso de re-consulta ospecha de dengue o diagnósticos errados)

Demora 2

Demora 3

(Tiempo) 
Se realizaron 37 entrevistas, de las cuales 21 fueron a casos y controles, y 16 a profesionales de la salud. En la mayoría de las entrevistas a casos y controles, la madre fue la persona que dio la información (19/21); en uno de los casos fue el padre y en uno de los controles, él mismo. De los 21 pacientes, solo uno no estuvo afiliado al sistema de salud. A continuación se presenta el análisis de cada una de las demoras y una representación gráfica a manera de síntesis, que ilustra los itinerarios de los casos (ver figura 1) y controles (ver figura 2).

\section{Demora 1. Conocimientos, aparición de los síntomas y cuidado inicial}

Se evidenció que las familias reconocen campañas de prevención y tienen información sobre dengue proveniente de medios de comunicación, avisos en instituciones de salud y folletos que hablan del control vectorial. Asocian las campañas a un problema relacionado con el control del mosquito: "pasan informaciones, volantes sobre controlar el dengue, pero uno hace caso omiso" (familiar de control).

En las familias aún se piensa en la fumigación como la estrategia más exitosa, y que es obligación de las autoridades. La mayoría de las familias de los casos y controles insistieron en que no vivían con el vector, y que sus seres queridos enfermaron fuera de sus casas. Las respuestas más reiterativas sobre cómo se transmite y cuáles son los síntomas del dengue fueron que es transmitido por un mosquito y que se comporta como una gripa fuerte, con fiebre, dolor de cabeza y dolor en los huesos.

A la pregunta sobre si tuvieron algún conocido con dengue, algunas personas no reconocieron alguien cercano que hubiese enfermado. Otras mencionaron que habían tenido dengue "suave", "sin gravedad", o que tenían familiares que habían enfermado, pero que desconocían que podía agravarse hasta la hospitalización o la muerte. "Uno cree que son virosis que están dando, que gripa... pero uno nunca imagina que un hijo llegue a presentar algo así de grave” (familiar de control); “yo sabía que era grave pero no que se iba a llevar a mi niña” (familiar de caso).

En las cinco ciudades, la mujer fue la cuidadora, y la persona que tomó las decisiones en salud de la familia. Mamás, tías y/o abuelas observaron el estado de salud de sus familiares evaluaron cambios, hicieron hipótesis sobre diagnósticos y observaron la evolución del enfermo: "Yo le tomaba la temperatura cada rato"; 
"parecía una virosis, pero yo estaba pendiente todo el tiempo de los síntomas y no dormía" (familiares de casos).

La aparición de fiebre y dolores de cabeza no generaron la búsqueda de atención médica inmediata en las familias, y fue generalmente al segundo día que decidieron asistir a los servicios de salud. Uno o más síntomas que persistieron (fiebre que no bajó, dolor de cuerpo, sangrado nasal, y/o vómito persistente) motivaron la búsqueda preocupada de atención en salud y la automedicación (que ocurrió en 10 de los 21 participantes). "El niño estaba demasiado ardido en fiebre y le dábamos acetaminofén y no le bajaba” (familiares de caso).

Además de estos síntomas, otra razón para tomar la decisión de buscar atención en salud fue cuando hubo una red de apoyo (familiares, vecinos) que ayudó con el cuidado de la casa y de otros miembros de la familia, aportando dinero o transporte y apoyando la decisión de buscar atención médica cuando las soluciones caseras no funcionaron.

A pesar que en muchas de las entrevistas se describió una mala percepción del sistema de salud por experiencias previas, la mayoría de los casos y controles asistieron oportunamente al servicio de salud y confiaron en el saber médico.

Yo primero pensé: para qué voy al hospital a esperar con la niña y a perder el día para que me den acetaminofén [...] pero después, ya viendo que la niña seguía mal, me preocupé y corrí para el médico, pues ellos son los que saben (familiar de caso).

\section{Demora 2. El recorrido en búsqueda de atención médica}

Una vez las familias salieron de la vivienda rumbo a una institución de salud, no tuvieron dificultades con los desplazamientos, la calidad del transporte ni de las vías. El medio de transporte utilizado dependió del grado de deterioro del caso o del control. El transporte más frecuente en la primera y segunda consulta fue el bus urbano, seguido de la moto, el taxi, la bicicleta y el recorrido a pié. No obstante, cuando se presentaron síntomas considerados por la familia como de mayor gravedad se utilizó el taxi (y solo en un caso, el vehículo particular) como medio de transporte.

Como punto importante de esta demora, los casos y controles no fueron al lugar de atención más cercano, sino al lugar asignado por el sistema de salud. Como se muestra en las figura 1 (síntesis de casos), los desplazamientos fueron de máximo 15 minutos, y en la figura 2 (síntesis de controles) de máximo 45 
minutos, con un costo máximo de 10.000 pesos (\$5,0 USD): "Fuimos en bus

a donde lo recibían con esa EPS (Empresa Promotora de Salud), como a 45 minutos con el tráfico"; "Pensábamos que era una gripa, una virosis, lo llevamos en la moto"; "él fue a donde lo atendían, como 40 minutos hasta allá en taxi" (familiares de casos y controles).

Con respecto a la demora 2 , fue común que las familias esperaran hasta el día siguiente para buscar atención en horas de la mañana, evitando desplazamientos en la mitad de la noche, a pesar de que todos los servicios de salud asignados funcionaban las 24 horas. Un caso y un control no consiguieron transporte en la noche por falta de disponibilidad de taxis o por inseguridad en el barrio.

\section{Demora 3. Ingreso, atención e itinerarios en el sistema de salud}

En cuanto a esta demora, se evidenció en las entrevistas fallas y problemas estructurales del sistema de salud colombiano, ejemplificados en la crisis financiera de varias de las instituciones de salud donde fueron atendidos los casos y controles, con problemas para cubrir honorarios del personal o fallas en la capacidad instalada: "llevan varios meses sin pagarnos y el lugar está en liquidación [...] a veces faltan algunos insumos, estamos esperando que se recupere financieramente" (profesional de salud). También fallas en el funcionamiento de la red pública en las ciudades, lo que obligó a esperas, re-consultas y desplazamientos de las familias entre instituciones: "la atención era muy mala [...] nos fuimos por nuestra cuenta para otro lugar" (control); "no hay una buena comunicación entre la baja y la alta complejidad, hay muchos problemas y todos se culpan entre si, y al final el paciente es el que sufre" (profesional de salud).

Otros ejemplos son la baja disponibilidad de ambulancias: "no había ambulancia, y tuvimos que esperar mucho, yo hubiese sabido y me hubiera llevado a la niña en taxi” (familiar de caso). Por otra parte, los profesionales narraron poca capacitación del personal, y una gran saturación de los servicios de urgencias:

[...] muchos profesionales hacemos turno en varias instituciones, y no hay mucho tiempo, lo mismo que pocos espacios de capacitación [...] el otro problema es que el servicio de urgencias siempre está lleno, y todo es de afán, hay un gran estrés en estos espacios y por más que se quiera, no se da abasto, además llega gente que está aburrida de consultar en la baja complejidad y vienen directamente a instituciones de mayor complejidad (profesional de salud). 
En los servicios de urgencias de las instituciones de salud, en su mayoría de baja complejidad, todos los entrevistados tuvieron que esperar entre 1 y 5 horas para ser atendidos. La narración de la espera en urgencias indicó que se daba prioridad a pacientes heridos o con aspecto visible de gravedad. En 18 de las 21 entrevistas, se evidenció que las fallas se concentraron en la baja complejidad de atención, pues fue en este escenario donde ocurrió la consulta o consultas iniciales fallidas, y que para las familias significó el agravamiento de los síntomas y la posibilidad de que su familiar empeorara o falleciera:

[...] yo creo que el error estuvo en (donde consultamos primero) acá en el barrio, pues si nos hubieran dicho qué era desde el principio y no hubiésemos tenido que ir varias veces, el niño no se nos muere [...] en el otro sitio de mayor complejidad si nos atendieron bien pero era demasiado tarde (familiar de caso).

Adicionalmente todas las narraciones (a excepción de la de un adulto que falleció en urgencias) indicaron que consultaron inicialmente en instituciones de menor complejidad, en las cuales fueron diagnosticados erróneamente o fueron remitidos a consulta externa. Allí fueron enviados a sus casas, donde se deterioraron y complicaron: "la doctora, dijo que tocaba sacar al niño por consulta externa, que a la gente le gustaba estar de paseo por el hospital" (familiar de caso).

Fueron usuales en las entrevistas ejemplos de una, dos, y hasta tres remisiones hacia otras instituciones, debido a la falta de cupo o camas, disponibilidad del servicio de Unidad de Cuidado Intensivo (UCI), o por temas de cobertura de la empresa de salud:

[...] entonces empezaron a llamar a otras instituciones porque ahí no había cama en la UCI [...] la doctora me decía que esperáramos a que llamaran de alguna institución donde hubiera cama para llevarlo [...]. Cuando al fin confirmaron en una institución que sí había cama, tocó esperar la ambulancia [...] al buen rato por fin salimos (familiar de caso).

Este proceso de remisión entre las instituciones de menor y mayor complejidad implica un ingreso formal al sistema de salud luego del proceso de re-consulta, donde las decisiones en cuanto al traslado y manejo de la persona enferma depende del personal de salud y de las condiciones logísticas del prestador del servicio (figuras 1 y 2). Además, en este punto el itinerario se empieza a alejar del entorno próximo del paciente, implicando mayores dificultades a los familiares en cuanto al transporte, ausencia laboral, alimentación y otras que se comentan a continuación. 
En las figuras 1 y 2 se presenta el proceso de re-consulta que fue similar para los casos y los controles, lo que implicó un regreso al hogar y luego volver a tomar la decisión de consultar teniendo en cuenta la información ya recibida de los profesionales de salud y la persistencia o empeoramiento de los síntomas. Igualmente, en las figuras se contrasta la diferencia entre los itinerarios donde los controles frecuentaron máximo 2 instituciones de salud, a diferencia de los casos que en su mayoría ( 9 de 13) frecuentaron 3 o 4 instituciones de salud durante el itinerario antes del fallecimiento.

Algunas familias cansadas de ser remitidas o de re-consultar decidieron cambiar de institución de salud a una de mayor complejidad por sí mismas:

[...] la llevé a una (institución de baja complejidad) y allá le aplicaron una inyección y le mandaron exámenes [...] y que no tenía nada. Pero a ella no se le quitaba la fiebre, entonces la lleve otra vez. Por la fiebre le dieron una inyección y nos mandaron a la casa. Esa noche ella empezó a vomitar, entonces me la lleve [...] y dijeron que era un daño de estómago [...] le mandaron dieta blanda y llevarla a los dos días [...] le dieron una inyección para el vómito y para la casa. Un día después, viendo que no había mejoría fuimos a una institución de mayor complejidad (familiar de caso).

El momento de agravamiento fue un momento de ruptura, en el que las familias evidenciaron que hubo mal servicio en el primer lugar de atención y lentitud en la atención para percatarse de la enfermedad. En algunos casos, tuvieron que oír profesionales hablando sobre la gravedad de su familiar: "escuchaba a los doctores en mi camilla, [...] empezaban a hablar entre ellos en clave pero uno siempre entendía [...] ese día me sentí súper mal, vi la muerte encima (control).

Una vez se llega a otra institución de salud de mayor complejidad, el nuevo interrogatorio y el tiempo de espera (en ocasiones en urgencias) para la reconfirmación del diagnóstico fue percibido por las familias como injustificado: "esperando para confirmar lo que ya sabíamos desde la primera institución, que era un dengue" (familiar de caso). Por otro lado, se narraron dificultades para acceder al servicio de ambulancia.

La llegada y acceso a la institución de mayor complejidad no fue dispendiosa en cuanto a distancias o trámites, pero para entonces las familias pudieron comparar la atención recibida en niveles de menor complejidad con la de niveles de mayor complejidad, resaltando que la atención fue mejor en estas últimas. Sin embargo, las entrevistas evidenciaron que hubo problemas de comunicación entre profesionales de salud y familiares: "Al niño lo mandaron a piso (habitación), y 
me tranquilizaron, pero luego viene una enfermera y me dice como si nada que lo iban a llevar a la UCI, yo quedé muy preocupada” (familiar de caso).

Los familiares (en especial las madres) se organizaron para asegurar los recursos suficientes para el transporte o la comida fuera de casa, regresar al hogar cada mañana para bañarse, comer algo, cuidar a los hijos en casa, preparar alimentos, trabajar de forma parcial, dejar el trabajo y volver al lugar de hospitalización:

Yo estaba trabajando en una empresa y me mandaron la carta de retiro porque no me podían tener trabajando así [...] me dieron una semana más de trabajo y me tocó salirme para estar pendiente de la niña (familiar de control).

Para 4 de los 8 itinerarios de controles indagados, el servicio más especializado a lo largo del itinerario fue la UCI, para los otros 4 hubo hospitalización regular. En cambio, 11 de los 13 casos fallecieron en la UCI, mientras los otros 2 fallecieron en hospitalización. Se reportó que la condición médica de los casos, según los familiares, era crítica al momento en que se confirmó la remisión a UCI. Adicionalmente, de los 11 casos que se remitieron a la UCI, 6 reportaron falta de cupo o disponibilidad de ambulancias para el traslado, a pesar de que eran recorridos cortos.

Al irse deteriorando el caso en la UCI, se presenta otra nueva ruptura, en la que las familias observaron el decaimiento de su familiar y asumieron la posibilidad de una mortalidad. En esta fase reportaron divergencias en la información entre profesionales de salud sobre la evolución del paciente: "uno me dijo que estaba mejorcito, [...] y al rato el otro me dijo que se iba a hacer lo imposible pero que esperara lo peor, por lo avanzado que estaba” (familiar de caso).

Una característica de varios itinerarios fue un momento de crisis, donde las personas dejan de creer que su familiar esté recibiendo una atención adecuada y surge una escena de reclamación o pelea que paradójicamente desemboca en una respuesta más ágil del sistema. En la visión de los familiares de casos y controles, un servicio negado que luego se hace efectivo después de una confrontación con los profesionales o administrativos, significa un servicio que depende de voluntades individuales y no de tener derecho a éste: "no me decían nada, esperé mucho, y con rabia le peleé a la enfermera y al médico y ahí si me atendieron” (control).

Hubo algunas divergencias entre la percepción familiar y la percepción de los profesionales de salud. Los familiares (en su mayoría de casos) opinaron que tuvieron que hacer varias re-consultas y que hubo mala atención, mucha espera, 
poca amabilidad y tiempo para atenderlos. Opinaron que hubo poca oportunidad

en temas como exámenes, remisiones a otra institución y traslados en ambulancia.

Por otro lado, algunos profesionales de salud comentaron que los reconsultantes saturan el sistema de salud y hay dificultades para la atención, primero por la gran extensión de la ficha de notificación, cuyo diligenciamiento es dispendioso en contraste con el poco tiempo de inter-consulta. También narraron inestabilidad laboral haciendo turnos en varias instituciones para completar un ingreso digno (cuando lo reciben puntualmente). Cuestionaron el tipo de contratación (como contratistas independientes), que ha llevado a la huelga en algunas instituciones, lo mismo que la falta de espacios de educación y actualización, así como dificultades para comunicarse y trabajar en equipo. Todos estos factores influyen desde la perspectiva de los profesionales entrevistados, en la prestación de un servicio oportuno, con calidad y acorde con el sistema de vigilancia en salud pública (profesionales de salud en las 5 ciudades).

\section{Análisis geográfico de las demoras}

Como se mencionó en el análisis de cada demora, desde una mirada espaciotemporal de los itinerarios, los diagramas síntesis de casos y controles (figuras 1 y 2) permiten identificar patrones repetitivos, momentos de ruptura o crisis que generaron cambios en el itinerario de las familias, al depender de las normas y acciones dictadas por profesionales de la salud, por el personal a cargo de trámites, y por el sistema de salud en general.

Se muestra que los eventos concernientes al acceso y calidad de los servicios de salud (demora 3) ocupan gran parte de los diagramas espacio-temporales, mientras que las demoras 1 y 2 no tuvieron tanta predominancia en los itinerarios. Sin embargo, el proceso de re-consulta en los diagramas sugiere una interdependencia entre las diferentes demoras, generando un movimiento no lineal entre las mismas. En el ir y venir entre el hogar y las instituciones de salud de menor complejidad se pierde tiempo que resulta en un empeoramiento de la situación y que implica un proceso de remisiones dentro del sistema de salud, hasta llegar a un desenlace (muerte o recuperación).

De igual forma, estos itinerarios no se pueden ver aislados y desconectados de itinerarios anteriores y futuros, considerando que el proceso no finalizó con la recuperación o la muerte en la institución de salud, sino que continuó con 
una serie de itinerarios posteriores que se enmarcan en un proceso que dentro la investigación denominamos como "proceso de retorno a la demora 1", como se menciona a continuación. Varias re-consultas en casos y controles repercuten en un impacto social y psicológico que influye en las acciones y concepciones de las personas para afrontar futuros itinerarios de salud.

\section{Proceso de retorno: demora 1 después de la muerte o la recuperación.}

En las entrevistas se identificaron otros aspectos que pueden configurar percepciones negativas de la calidad del sistema, relacionadas con el proceso de atención. Las familias no reconocen seguimiento después del evento, ni haber recibido información adicional a la que ya sabían: "tal vez si visitaron pero no dijeron nada nuevo, de pronto una revisión del tanque o fumigar la cuadra, o un folleto, pero no preguntan cómo siguió el niño cuando casi se muere" (familiar de control).

Sobre la necropsia, las familias comentaron que entienden su obligatoriedad, pero que hubo poca comprensión de los profesionales para entender el duelo al informar sobre los fines del procedimiento. Después del ritual de las exequias, las familias de los casos deben retornar a "la normalidad". Este "retorno" a la primera demora lo sigue acentuando el sistema, que lleva a cabo visitas, encuestas y control vectorial, a veces sin tacto: "vienen y me preguntan cómo seguía el niño, cuando hacía una semana que había muerto” (familiar de caso).

En dos entrevistas se reportó una visita posterior de una psicóloga encargada de la asesoría y seguimiento a familiares de casos. Para las familias significó la presencia de personal sin experiencia en el manejo del duelo y de la enfermedad: "Si, acá vino una psicóloga a hablar con nosotros, pero era una joven que no sabía mucho de la enfermedad [...] estaba nerviosa, nosotros terminamos haciéndole terapia a ella (risas)" (familiares de caso).

De forma adicional, 6 familiares de casos presentaron recursos legales contra el sistema de salud, y los demás pensaron en hacerlo, sin embargo, quienes demandaron debieron pagar un abogado, y asumieron trámites, citaciones, búsqueda de datos en historias clínicas, que fueron un gasto y barrera adicional. Todos estos factores presentes en este itinerario hacen que las familias decidan no seguir adelante con este tipo de recursos legales. 
Se evidenció también en este retorno un cambio de discurso en los casos y controles, relacionado con la práctica de la automedicación. Más que declarar que no debían administrar medicamentos a sus familiares enfermos, las familias (y en especial las mamás) se mostraron reflexivas sobre administrar medicamentos como ácido acetilsalićlico, ibuprofeno o antigripales a otros familiares con cuadros febriles.

Este "retorno" a la demora 1 estaría relacionado entonces con procesos como la necropsia, las visitas de seguimiento institucional, las demandas y la ausencia de apoyo psicosocial, que configuran un discurso negativo hacia el sistema, y la idea de acceder a una atención efectiva mediante un nivel de confrontación mayor y más insistencia. Así, la mayoría de las personas entrevistadas comentaron cómo, ante la negación de una serie de servicios, la constante fue el testimonio de "tuve que pelear o tuve que asumir costos” (este más asociado a la falta de ambulancias).

\section{Discusión}

Esta investigación, permitió identificar las principales demoras en los casos y controles. En cuanto la demora 1 se identificó que la mayoría de ellos conocen o han escuchado sobre la enfermedad y reconocen los síntomas asociados. Sin embargo, estos síntomas no generaron búsqueda de atención inmediata. Solamente con el sangrado, el brote, el vómito o la persistencia de la fiebre se tomó la decisión de consultar a las instituciones de salud. No se identificaron problemas asociados a barreras de tipo económico o de género que impidieran la toma de decisión por parte de los familiares.

En cuanto a la demora 2, no se identificaron barreras geográficas o asociadas a falta de recursos para el transporte, pero el diseño del sistema de salud no permitió que las personas consultaran en el lugar más cercano si no en el lugar asignado. Esta demora no se presentó como un problema en la medida que los tiempos y gastos no fueron elevados, y existía amplia oferta de transporte, por lo que se logró sortear las dificultades que surgieron. Adicionalmente operaron redes de apoyo que permitieron sobrellevar la falta de recursos para el transporte.

En la demora 3, se evidenciaron procesos como la re-consulta porque los pacientes continuaron enfermos y se deterioraron a pesar de haber consultado a los servicios de salud, así como dificultades en la comunicación médico-paciente, 
y demoras en los procesos de remisión a otros niveles de mayor complejidad. Igualmente, se registraron problemas relacionados con la estabilidad laboral de los profesionales de salud. Por otro lado, los casos consultaron más instituciones (hasta 4 diferentes), que los controles (hasta 2 diferentes). En general, los entrevistados percibieron una deficiente calidad de la atención en las instituciones de baja complejidad en comparación con los de mayor complejidad. Con la experiencia, los casos recordaron nombres de profesionales de salud, resultados de exámenes, fechas y horas exactas de forma vívida y clara. En los controles el momento más crítico fue la entrada a la UCI, y el aprendizaje fue que el dengue se entendió como mortal, muy grave, inesperado, doloroso y peligroso.

Al hacer un contraste entre el número de instituciones donde asistieron los casos y controles (sea por remisión del sistema o porque re-consultaron por voluntad propia), hay una marcada diferencia entre los que se recuperaron y los que fallecieron. De los controles, ninguno pasó por más de 2 instituciones de salud, mientras que la mayoría de los casos ( 9 de 13) pasaron por tres o cuatro instituciones. Un hallazgo claro es que entre más tiempo transcurrió en el proceso de búsqueda de atención y más instituciones se visitaron, hubo mayor riesgo de morir, lo que se evidencia en el hecho de que las personas fallecidas consultaron un número mayor de instituciones que aquellas que se recuperaron.

Desde la geografía del tiempo se considera que los movimientos de las personas en el tiempo y el espacio no son al azar. Por el contrario, se considera que hay unas restricciones que condicionan estos recorridos y que en últimas son un reflejo de las estructuras sociales (HÄGERSTRAND, 1970). Los aportes de esta investigación permiten una perspectiva transdisciplinar en el análisis de las demoras y el dengue, y la posibilidad de encontrar matices en el proceso de búsqueda de atención que no son reconocidos por el sistema de salud.

La mayoría de los análisis sobre demoras en la literatura (WALKER et al., 1986; CASTELLANOS, 1990; THADDEUS; MAINE, 1994; BOBADILLA; REYES-FRAUSTO; KARCHMER, 1996; BARNES-JOSIAH; MYNTTI; AUGUSTIN, 1998; FANEITE, 2006; PHYSICIANS FOR HUMAN RIGHTS, 2007; FERNÁNDEZ et al., 2009; RODRÍGUEZ-ANGULO et al., 2012; WAISWA et al., 2010; VEGA, 2012; UPADHYAY; RAI; KRISHNAN, 2013) concluyen con la ocurrencia de la mortalidad. En solo uno se resaltó y reconoció el factor emocional, el impacto social y la atención psicológica para el 
manejo del duelo, como factores a tener en cuenta después de una mortalidad (PITARQUE et al., 2007).

Mazza y Vallejo (2012) anotan que los resultados de varios estudios sobre demoras presentan problemas en la realización de la autopsia verbal o interrogatorio a familiares y pacientes (MAZZA; VALLEJO; GONZÁLEZ, 2012). Se resalta en esta investigación que la entrevista y el análisis espacio-temporal de itinerarios, complementa herramientas como la autopsia verbal y la reciente autopsia social (KALTER et al., 2011), permitiendo una mayor profundidad y comprensión del proceso de búsqueda de atención médica. La reconstrucción de los itinerarios de forma gráfica, permitió reconocer la no-linealidad y lo cíclico de las demoras, visibles en la complejidad de los itinerarios y el proceso de retorno a la demora 1.

Al discutir la linealidad de las demoras (que plantea en la mayoría de estudios un proceso de enfermedad-recorrido-atención-mortalidad), emergió que el proceso de búsqueda de atención se puede comportar de forma cíclica, pues una vez concluye el itinerario con la recuperación o la muerte empieza luego un nuevo ciclo de búsqueda de atención con otra o la misma patología.

El margen de actuación de estas familias frente a las fallas del sistema de salud está limitado por la historia natural de la enfermedad, haciendo que se presenten demandas cuando el paciente ya ha fallecido. Los recursos legales (derechos de petición, tutelas) y los itinerarios juegan un papel fundamental para entender las lógicas y restricciones impuestas por el sistema de salud, como lo amplían Abadía y Oviedo (2009) al plantear los itinerarios burocráticos que operan para el acceso a salud con calidad en Colombia (ABADÍA; OVIEDO, 2009).

La aproximación cualitativa permitió evidenciar las tensiones y posibilidades de mejora propuestos a partir de la experiencia de todos los actores. Más allá de culpabilizar a los pacientes o al personal de salud, estos abordajes permiten describir de forma más profunda el rol de los actores involucrados en el proceso de atención, como sujetos con derechos y con la posibilidad de generar cambios y exigencias al sistema de salud.

\section{Conclusiones}

A partir de los itinerarios vividos por los familiares de casos y controles, se puede cuestionar lo que se entiende como la exigibilidad del derecho a la salud. Las personas asumen estrategias para acceder a una atención de calidad, acudiendo a 
la discusión y confrontación con las instituciones de salud, las cuales responden con un mensaje equívoco reforzando la idea que peleando es la única forma de lograr una atención médica efectiva.

Se identificó la necesidad de formación continuada de profesionales de salud en zonas endémicas para la identificación adecuada de los signos y síntomas del dengue. Se destaca la pertinencia del seguimiento telefónico a los pacientes y la importancia de mejorar la notificación al Sistema de Vigilancia Epidemiológica.

La investigación debe avanzar en la identificación de los momentos de tensión visibles en los itinerarios de las personas, incluyendo enfoques que valoren la relación entre el ser humano, su entorno social y ambiental, y la salud (CHARRON, 2012), profundizando en las fuerzas estructurales y complejas que los generan. Se recomienda proponer de forma transdisciplinar estrategias de mejoramiento de la calidad de la atención en dengue (y otras patologías) con participación de todos los actores, que trasciendan de la mirada simplista de culpabilizar al otro, y reduzcan el impacto de epidemias. Estos resultados pueden ser insumo para la toma de decisiones en la búsqueda de una reducción de la mortalidad por dengue en las cinco ciudades. ${ }^{1}$

\section{Limitaciones en el estudio}

Durante la recolección de la información hubo dificultades para realizar algunas entrevistas, dado que los familiares de varios casos se negaron a participar argumentado "duelo", "molestia hacia el sistema de salud", o "no tener intención de narrar su experiencia”.

\section{Agradecimientos}

A las familias que amablemente participaron de las entrevistas. A las instituciones, funcionarios, profesionales de salud, y demás personas en las cinco ciudades que facilitaron la investigación. A COLCIENCIAS, convocatoria 537/2011.

\section{Bibliografía}

ABADÍA, C. E.; OVIEDO, D. G. Bureaucratic itineraries in Colombia. A theoretical and methodological tool to assess managed-care health care systems. Social Science \& Medicine, v. 68, n. 6, p. 1153-1160, 2009. 
BARNES-JOSIAH, D.; MYNTTI, C; AUGUSTIN, A. The "three delays” as a framework

for examining maternal mortality in Haiti. Social Science \& Medicine, v. 46, n. 8, p. 981993, 1998.

BELTRAMIRO, F. G. Metodologías cualitativas en ciencias sociales: modelos y procedimientos de análisis. Buenos Aires: Editorial Biblos, 2004.

BOBADILLA, J.; REYES-FRAUSTO, S.; KARCHMER, K. Magnitud y causas de mortalidad materna en el Distrito Federal (1988-1989). Gac Med Mex, v. 132, p. 5-16, 1996.

CASTELlanOS, L. D; VASQUEZ, J. O; DAVID, V. Mortalidad de mujeres en edad reproductiva y mortalidad materna. Universidad Nacional Autonoma de Honduras Facultad de Ciencias Medicas, 1990.

CHARRON, D. F. Ecohealth research in practice. New York: Springer, 2012.

FANEITE, P. Mortalidad materna y perinatal. Tendencias 1969-2004. Rev Obstet Ginecol Venez, v. 66, n. 2, p. 75-79, 2006.

FERNÁNDEZ, J. A. et al. Caracterización de la mortalidad por malaria en el Valle del Cauca, 2005-2006. Biomédica, v. 29, n. 4, p. 582-590, 2009.

HÄGERSTRAND, T. What about people in regional science? Papers Reg Scienc Assoc, v. 24, n. 7, p. 21, 1970.

KALTER, H. D. et al. E. Social autopsy for maternal and child deaths: a comprehensive literature review to examine the concept and the development of the method. Popul Health Metr, v. 9, p. 45, 2011.

MAINE, D. et al. Diseño y evaluación de programas para mortalidad materna. New York: Columbia University, 1997.

MARTÍNEZ TORRES, E. Dengue. Estudos avançados, v. 22, n. 64, p. 33-52, 2008.

MAZZA, M. M.; VALLEJO, C. M.; GONZÁLEZ BLANCO, M. Mortalidad materna: análisis de las tres demoras. Rev. obstet. ginecol. Venezuela, v. 72, n. 4, p. 233-248, 2012.

ORGANIZACIÓN PANAMERICANA DE LA SALUD. Guías para la atención de enfermos en la región de las Américas. La Paz: PAHO, 2010.

PACAGNELLA, R.; CECATTI, J. The role of delays in severe maternal morbidity and mortality: expanding the conceptual framework. Reproductive Health Matters, v. 20, n. 39, p. $155-163,2012$.

PADILlA, J. C.; ROJAS, J. C.; GÓMEZ, R. Dengue en Colombia: epidemiología de la reemergencia a la hiperendemia. Bogota: Guías de Impresión, 2012.

PHYSICIANS FOR HUMAN RIGHTS. Demoras fatales, mortalidad materna en el Perú: un enfoque desde los derechos humanos para una maternidad segura, 2007. Washington. Obtenido de: http://www.bvcooperacion.pe/biblioteca/handle/123456789/2216. Acceso en: 1 jun. 2014. 
PITARQUE, R. et al. Estudio de la mortalidad infantil en un municipio de la Provincia de Buenos Aires 2003-2007, 2007. Disponible en: http://www.medicinaysociedad.org. ar/publicaciones/01_DIC_2010/ESTUDIO\%20DE\%20LA\%20MORTALIDAD\%20 INFANTIL\%20EN\%20UN\%20MUNICIPIO\%20DE\%20LA\%20PROVINCIA\%20 DE\%20BUENOS\%20AIRES.pdf. Acceso en: 10 may. 2014.

RODRÍGUEZ-ANGULO, E. et al. Demoras en la atención de complicaciones maternas asociadas a fallecimientos en municipios del sur de Yucatán Mexico. Rev Biomed, v. 23, p. 23-32, 2012.

THADDEUS, S; MAINE, D. Too far to walk: maternal mortality in context. Social science \& medicine, v. 38, n. 8, p. 1091-1110, 1994.

UPADHYAY, R. P.; RAI, S. K.; KRISHNAN, A. Using three delays model to understand the social factors responsible for neonatal deaths in rural Haryana, India. Journal of tropical pediatrics, v. 59, n. 2, p. 100-105, 2013.

VEGA, L. Informe Final del Evento Dengue Año 2012. Bogotá, Instituto Nacional de Salud, 2012.

WALKER, G. A. et al. Maternal mortality in Jamaica. The Lancet, v. 327, n. 8479, p. 486488, 1986.

WAISWA, P. et al. Using the three delays model to understand why newborn babies die in eastern Uganda. Tropical medicine \& international health, v. 15, n. 8, p. 964-972, 2010.

\section{Nota}

${ }^{1}$ F. Ardila recolectó información, participó en el análisis e interpretación de resultados. S. Martínez concibió el diseño del estudio y su protocolo, recolectó información, participó en el análisis e interpretación de resultados, realizó revisión crítica del contenido del documento. M. Fuentes redactó la primera versión del documento, participó en el análisis e interpretación de resultados. E. Borrero concibió el diseño del estudio y su protocolo, participó en el análisis e interpretación de resultados, realizó revisión crítica del contenido del documento. 
Análise das demoras no atendimento em saúde em pessoas gravemente doentes ou que morreram de dengue em cinco cidades na Colômbia

Objetivo: Identificar demoras no atendimento em saúde de pessoas que faleceram ou se recuperaram de dengue grave em cinco cidades da Colômbia. Materiais e métodos: Estudo qualitativo com pessoas que faleceram em decorrência da dengue (casos) ou que se recuperaram (controles). Entrevistaram-se familiares e analisaramse seus itinerários durante o processo de procura de atendimento nos serviços de saúde. Os dados foram analisados baseados no modelo das "três demoras", complementado com diagramas espaço-temporais e entrevistas com profissionais de saúde. Resultados: Ainda que os signos e sintomas fossem desconhecidos, procurou-se atendimento rapidamente. $\mathrm{O}$ percurso para o acesso à primeira consulta não apresentou dificuldades. Apresentaram-se erros na qualidade do diagnóstico e identificou-se maior número de instituições consultadas em casos do que em controles. Evidenciouse um processo de retorno à demora 1 , fato que pode influir negativamente em decisões futuras. Conclusões: Complementar a análise clássica de demoras com a análise espaço-temporal de itinerários permite identificar falhas e acertos na qualidade do atendimento para a tomada de decisões.

Palavras-chave: dengue; cuidados médicos; mortalidade; análise qualitativa. 


\section{Abstract}

Analysis of delays in healthcare in people seriously ill or dead from dengue in five cities in Colombia

Objective: Identify delays in receiving healthcare in cases of patients who either recovered from or died due to severe dengue in five Colombian cities. Method: Qualitative study with deceased persons from dengue (cases) or that recuperated (controls). Family members were interviewed and their itineraries were analyzed. The data was analyzed from the "three delay" model, complemented with spatiotemporal diagrams and interviews to health professionals. Results: Despite not knowing signs and symptoms, attention was rapidly sought. There were no difficulties accessing the route to the first consultation There were flaws in the quality of diagnosis and a higher number of institutions were consulted in cases than in controls. It was evidenced a return to delay 1 that could negatively influence future health decisions. Conclusions: Complementing the delay analysis with spatiotemporal analysis of the itineraries permits the visualization of flaws and successes in the quality of care for decision-making.

> Key words: dengue; medical care; mortality; qualitative analysis. 